\title{
Measurement of Jet Cross Section and Fragmentation of Jets Using Tracks with the ATLAS Detector
}

Seth Zenz ${ }^{*}$ on behalf of the ATLAS Collaboration

UC Berkeley and LBNL, Berkeley, CA 94720, USA

E-mail: SCZenz@lbl.gov

\begin{abstract}
Jets are identified and their properties studied using tracks measured by the ATLAS Inner Detector. Events are selected using a minimum-bias trigger, allowing the study of jets at low transverse momentum to be done independently of the calorimeter. Jets are reconstructed using the anti- $k_{t}$ algorithm applied to tracks with two parameter choices, 0.4 and 0.6. An inclusive jet transverse momentum cross section measurement from $4 \mathrm{GeV}$ to $80 \mathrm{GeV}$ is shown, integrated over $|\eta|<0.57$ and corrected to charged particle-level truth jets. The probability that a particular particle carries a given fraction of the jet momentum (fragmentation function) is also measured. Comparison with the models implemented in existing tunings of Monte Carlo event generators with the data indicates reasonable overall agreement between data and Monte Carlo.
\end{abstract}

35th International Conference of High Energy Physics - ICHEP2010,

July 22-28, 2010

Paris France

${ }^{*}$ Speaker. 
QCD predicts the existence of high $p_{T}$ jets in proton-proton collisions. The emergence of these jets from the soft interactions of low-energy pp scattering indicates the momentum scale at which the collision can be described perturbatively. Jets are identified using charged tracks reconstructed in the ATLAS detector [1] at the LHC, and jet momentum and fragmentation distributions are corrected to the level of charged particle jets. The properties of these charged particle jets are not comparable to perturbative QCD calculations; however, they are compared to the phenomenological models of jet production and fragmentation that are implemented in Monte Carlo generators.

The properties of charged particle jets are investigated for $p_{\mathrm{T}}>4 \mathrm{GeV}$ and $|\eta|<0.57$. Charged particle jets are formed by applying the anti- $k_{t}$ jet algorithm [2] with parameter $\mathrm{R}=0.4$ or 0.6 to all primary charged particles (mean lifetime $\tau>0.3 \times 10^{-10} \mathrm{~s}$ ) with transverse momentum $p_{\mathrm{T}}>500$ $\mathrm{MeV}$. Two quantities are measured for these charged particle jets: the inclusive cross section as a function of momentum, and the jet fragmentation variable $z_{\text {track }}$, defined to be the component of each charged particle momentum along the jet axis, divided by the total jet momentum.

This analysis uses data from an initial period of ATLAS running with total luminosity of about $370 \mu \mathrm{b}^{-1}$. Events are required to have a reconstructed primary vertex. The efficiency of both the trigger and vertexing were determined from data to be negligibly different from $100 \%$. In these events, tracks are reconstructed using the silicon-seeded tracking algorithm [3]. They are required to have transverse momentum $p_{\mathrm{T}}>500 \mathrm{MeV}$, transverse and longitudinal impact parameters consistent with the primary vertex, and to pass minimum silicon hit requirements. Track jets are formed by applying the jet algorithm to tracks; the cross section and $z_{\text {track }}$ measured for the track jets are then corrected (unfolded) to truth-level charged particle jets. Events generated with the ATLAS MC09 [4] tune of Pythia 6 [5] with a GEANT 4 [6] model of the ATLAS detector are used to derive the corrections. Jet reconstruction efficiency and bin-to-bin migrations in the inclusive jet cross section are corrected via the Bayesian Iterative Unfolding algorithm [7]. For the fragmentation measurement, bin-by-bin correction factors are computed from Monte Carlo and applied to the data. Systematic uncertainties include tracking efficiency and fragmentation/underlying event, uncertainties associated with mismeasured high- $p_{\mathrm{T}}$ tracks, mismodelling of tracking efficiency in $\phi$, and an overall $11 \%$ luminosity uncertainty (on the cross section only) [8].

Cross sections are shown in Fig. 1 for $\mathrm{R}=0.4$ and 0.6, and compared to Monte Carlo generators and tunes described in [8]. Phojet agrees with these data within the systematic error, while the other tunes disagree by at least $40 \%$ in at least one bin. The fragmentation is shown in Fig. 2 for $\mathrm{R}=0.6$ for jet $p_{\mathrm{T}}$ in the $4-6$ and $10-15 \mathrm{GeV}$ ranges; plots for $6-10$ and $15-24 \mathrm{GeV}$ and for $\mathrm{R}=0.4$ are available [8]. For both R values, the ATLAS AMBT1 [9] tune of Pythia 6 agrees within systematic errors for jet $p_{\mathrm{T}}>10 \mathrm{GeV}$, while the other tunes disagree by at least $20 \%$ in at least one bin.

\section{References}

[1] ATLAS Collaboration, G. Aad et al., JINST 3 (2008) S08003.

[2] M. Cacciari, G. P. Salam, and G. Soyez, JHEP 04 (2008) 063.

[3] T. Cornelissen et al., J. Phys. Conf. Ser. 119 (2008) 032014.

[4] A. Buckley, H. Hoeth, H. Lacker, H. Schulz, and J. E. von Suggern, Eur. Phys. J C65 (2010) 331.

[5] T. Sjorstrand, S. Mrenna, and P. Skands, JHEP 05 (2008) 026. 


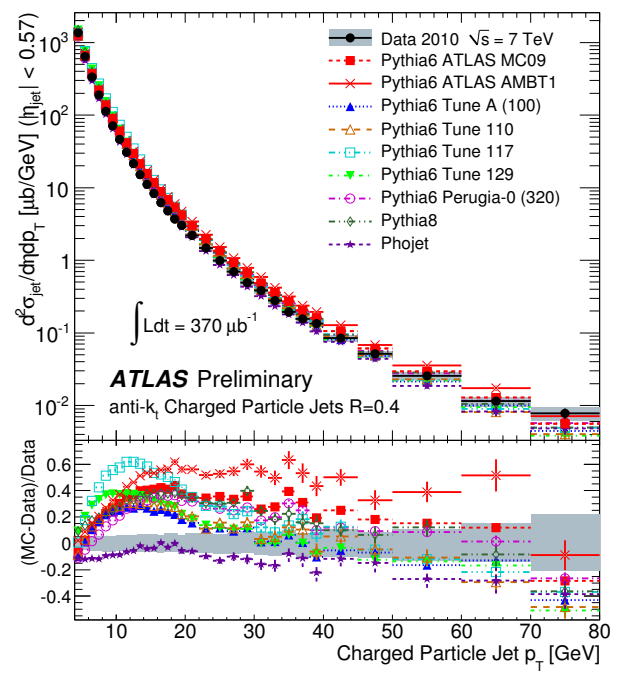

(a) $\mathrm{R}=0.4$

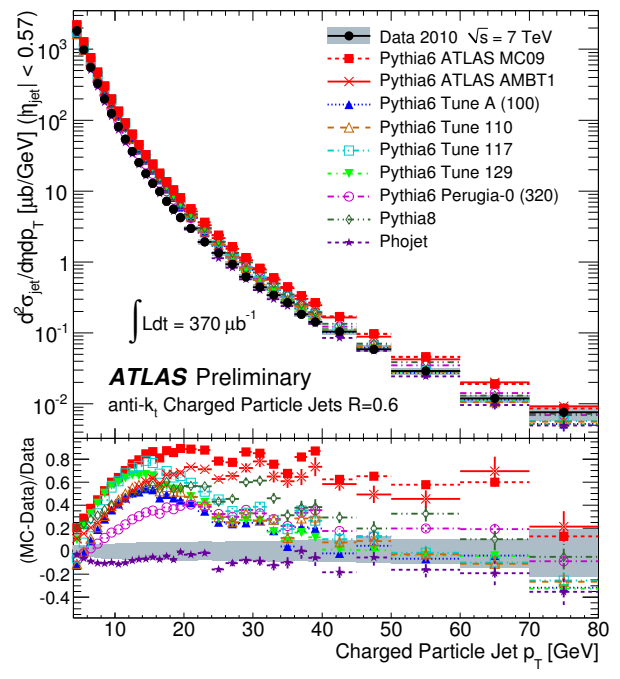

(b) $\mathrm{R}=0.6$

Figure 1: Corrected jet cross section for anti- $k_{t}$ jets, compared with event generator predictions. The shaded area is the total uncertainty for the corrected data distribution, excluding the $11 \%$ luminosity uncertainty.

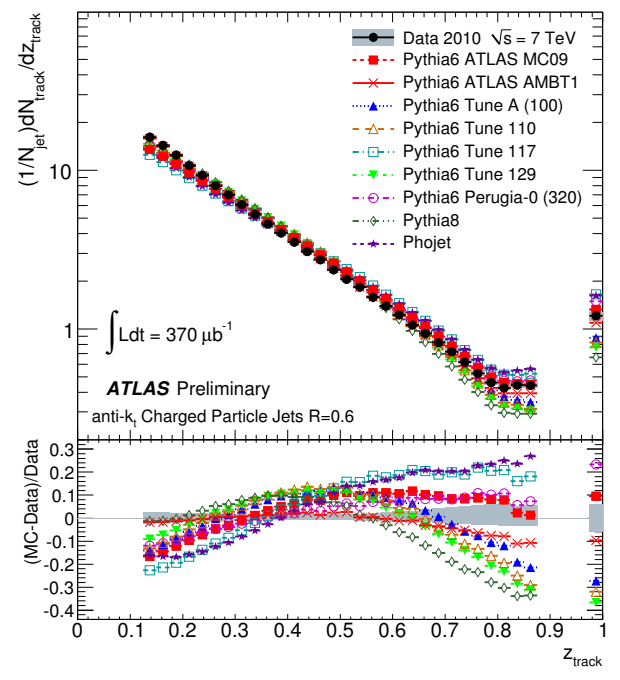

(a) $4 \mathrm{GeV}-6 \mathrm{GeV}$

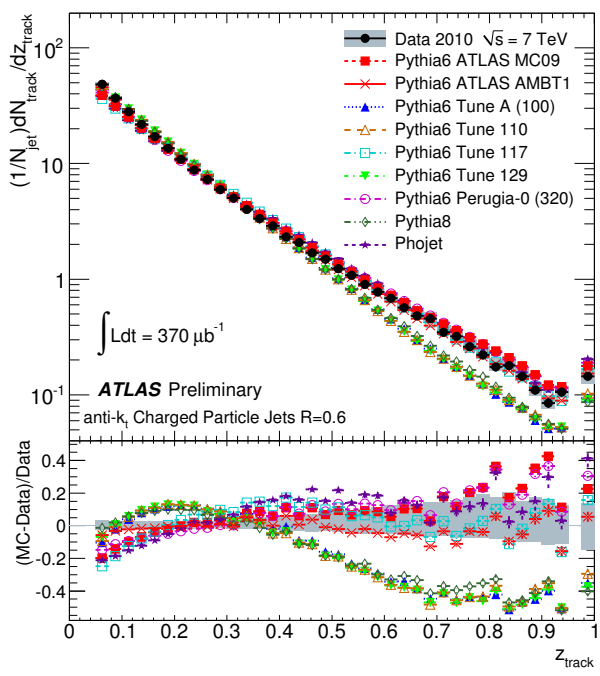

(b) $10 \mathrm{GeV}-15 \mathrm{GeV}$

Figure 2: Corrected fragmentation function in anti- $k_{t}$ jets with $\mathrm{R}=0.6$ in ranges of charged jet $p_{\mathrm{T}}$, compared with event generator predictions. The shaded area is the total uncertainty for the corrected data distribution.

[6] S. Agostinelli et al., Nucl. Instrum. Meth A506 (2003) 250.

[7] G. D’Agostini, Nucl. Instrum. Meth. A362 (1995) 487-498.

[8] ATLAS Collaboration, ATLAS-CONF-2010-049.

[9] ATLAS Collaboration, ATLAS-CONF-2010-031. 